\title{
Good Preclinical Bioanalytical Chemistry Requires Proper Sampling from Laboratory Animals: Automation of Blood and Microdialysis Sampling Improves the Productivity of LC/MSMS.
}

\author{
Fuming Xie, Craig S. Bruntlett, Yongxin Zhu, Candice B. Kissinger, and Peter T. Kissinger ${ }^{\dagger}$ \\ Bioanalytical Systems, Inc. (BASi), 2701 Kent Avenue, West Lafayette, IN 47906-1382, USA
}

\begin{abstract}
The preclinical bioanalytical process with animal models begins with sampling biological fluids and tissue. The goal is to understand oral absorption kinetics, distribution, metabolism, excretion, blood brain barrier penetration, drug-drug interactions, and the influences on biomarkers, hematology, electrophysiology, cardiology, blood pressure and behavior. An overview is obtained by periodic blood sampling of $8-12$ samples over a total time span of $10-24 \mathrm{~h}$. Urine, feces, bile and microdialysates can augment the information available from whole blood. In today's preclinical environment, the majority of samples are processed by LC/MSMS augmented by robotic sample preparation tools. These tools save labor and improve precision for smaller volume/lower concentration samples. Our laboratories have been engaged in a project that is focused on improving both the quality and throughput for laboratory animal studies, while providing for reduced numbers of animals and enhanced animal comfort. We have implemented a robotic system that can accomplish most of the above goals for laboratory rats, dogs and primates. Studies with mice are at an earlier stage, but feasibility has been demonstrated. This presentation is a progress report on this evolving research program in cooperation with multiple pharmaceutical and drug development companies. We will illustrate results and discuss future directions.
\end{abstract}

(Received January 31, 2003; Accepted February 14, 2003)

\begin{tabular}{|c|c|c|c|}
\hline 1 Introduction & 479 & 2.2 Automated blood sampling & \\
\hline 1.1 Animal science and analytical science are & & $2 \cdot 3$ Catheter details & \\
\hline $\begin{array}{l}\text { mutually dependent. } \\
2 \text { Sampling and Sample Preparation }\end{array}$ & 480 & 3 Results & $\begin{array}{l}483 \\
484\end{array}$ \\
\hline $\begin{array}{l}2 \text { Sampling and Sample Preparation } \\
2 \cdot 1 \text { Membrane sampling probes }\end{array}$ & & $\begin{array}{l}4 \text { Conclusion } \\
5 \text { References }\end{array}$ & 485 \\
\hline
\end{tabular}

\section{Introduction}

1.1 Animal science and analytical science are mutually dependent.

The goal of our laboratory is to provide better quality pharmacokinetic and pharmacodynamic data in a shorter period of time. Better data in less time is a constant goal of the pharmaceutical industry. Experiments performed in parallel rather than in series are a key ingredient. Following these principles reduces the number of animals required to arrive at a conclusion by refining the experimental protocols.

This is made possible by using advanced instrumentation and software to sample animals in a manner that dramatically reduces pain and stress. In fact, both are virtually eliminated $v s$. conventional techniques such as tail vein sampling and suborbital bleeds. It was an earlier advance to confine cannulated animals in restrainers after periodically transferring them from a home cage for blood draws. Pain is eliminated by the use of catheters, however, stress from the animal-human interaction remains substantial and the process is very labor intensive.

\footnotetext{
$\dagger$ To whom correspondence should be addressed

E-mail: pete@bioanalytical.com
}

With automation, it is straightforward to monitor behavior, collect urine and feces and carry out microdialysis sampling to monitor neurotransmitter or glucose response to experimental drugs. It is possible to sample from two catheters simultaneously to assess first pass effects and to even collect bile as well.

One of the reasons traditional methods of animal sampling (including euthanasia) became the standard prior to 1990 was that bioanalytical methods were quite crude, requiring large volumes (mL). Everything has changed in recent years. LC/MSMS is especially helpful for determination of drug candidates in both smaller volumes and at lower concentrations. This means that serial sampling of biological fluids over a range of volumes of $5-150 \mu \mathrm{L}$ per sampling point is very feasible in many cases. The low end of the range is key for mice and the high end is very practical for rats, hamsters or guinea pigs. Naturally, one can easily move to larger volumes for larger animals such as dogs or humans and take many serial samples with no measurable physiological stress. A key issue for the pharmaceutical industry is the amount of experimental drug available for the first animal studies. The progression - mouse $(25-30 \mathrm{~g})$, rat $(200-300 \mathrm{~g}), \mathrm{dog} / \mathrm{monkey}(5000-10000 \mathrm{~g})$, pig $(10000$ - $50000 \mathrm{~g})$, human $(60000$ - $80000 \mathrm{~g})$ - nicely illustrates why rodents are a good first step.

It is not helpful to invest a lot of money in bioanalytical 


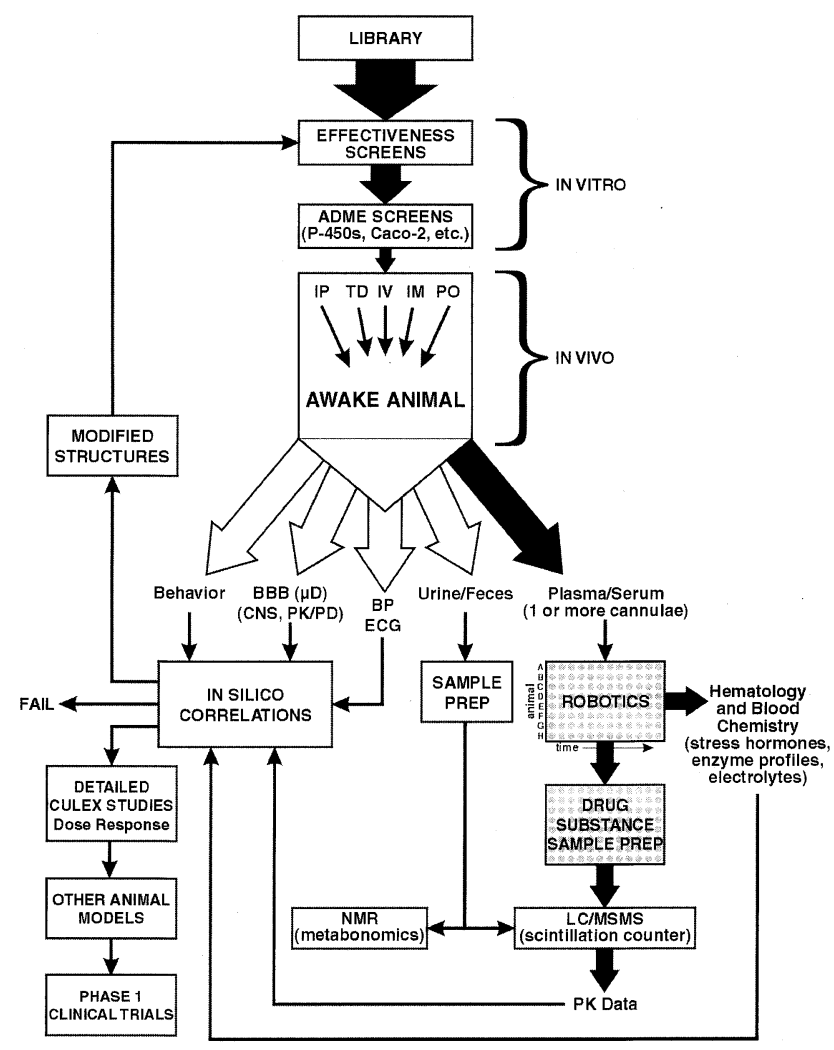

Fig. 1 How an automatic blood sampler (ABS) can fit into the late stages of discovery and all phases of preclinical development. Routes of administration: IP, intraperitoneal; TD, transdermal; IV, intravenous; IM, intramuscular; PO, per oral.

chemistry and then stress the animals to a point where the data is of very little value. Likewise, it is not helpful to improve the animal science and then neglect the bioanalytical protocols necessary to obtain quality data. Professionals from both areas understand this. Better animal welfare results in better science, and better analytical chemistry allows for reduced sample volumes. Likewise, experiments should be coordinated so that the samples are processed quickly and do not sit in freezers for many weeks. Only then can the full benefits of rapid decision making be realized.

\section{Sampling and Sample Preparation}

Sampling or obtaining a "representative sample" has long been a topic for courses in analytical chemistry. But how should an animal be sampled? Where and when? How many animals? In this article, we include animal sampling as a necessary consideration for quality analytical data.

Figure 1 illustrates the concept of coupling parallel animal science experiments with bioanalytical chemistry and software to facilitate drug development. The focus of this review is on fully functioning animal models of human physiology/pharmacology. The discovery phase of pharmaceutical research involves synthesizing libraries and screening them in vitro for effectiveness, metabolism and potential toxicity. Following those screens, a much-reduced number of compounds will have to be tested in complete mammalian species because it remains true that an animal is more than the sum of its parts. The pharmaceutical industry

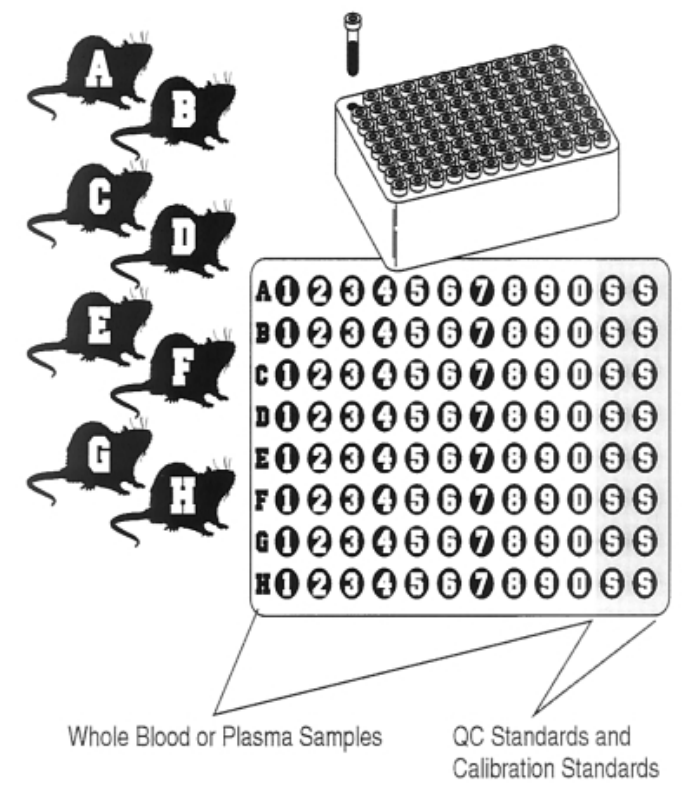

Fig. 2 All of the samples for PK screen using eight animals can be placed in $300 \mu \mathrm{L}$ sealed vials on one 96-well plate.

does not yet have full confidence in integrating in vitro data or using predictive software. Animal studies remain of primary importance, but they are expensive and have not until recently provided much opportunity for automation. As shown in Fig. 1, our goal was to be able to place animal samples in 96 well plates where there have already been many investments in sample preparation for LC/MSMS as well as other analytical methods. Figure 2 illustrates this further for a typical pharmacokinetics screening experiment using eight rats.

Once the samples are in the plate format, they can be centrifuged and transferred (Fig. 3). It is easy to add fluids to the plates, shake them and extract them with immiscible solvents (Fig. 4) or by solid phase extraction (Figs. 5 and 6). There are excellent 96 well autosamplers for chromatography that can manage multiple well plates for completely automatic processing overnight. The automation is much less expensive than human labor on a per sample basis and is now very reliable. Virtually all pharmaceutical research laboratories have implemented this sample preparation automation since the mid1990s. Universities have been slower to use this approach to bioanalytical chemistry because the number of samples is frequently much smaller and therefore utilization of the robotics is not as well justified.

Going back to Fig. 1, we can see the many roles for analytical chemistry. Not only is it important to monitor drugs and their metabolites in blood, but there is also much work to be done looking at the response of endogenous marker substances to the presence of the experimental drug. There are also samples of urine and feces to examine. In some cases radio labeled compounds are followed by LC with a radiochemical detector. High resolution NMR is beginning to be applied with urine as an early indicator of potential toxicology using an approach described as metabonomics..$^{1,2}$ Membrane sampling in specific organs such as brain or lung can provide samples parallel to the blood samples for pharmacokinetics (see following section). The dosing formulation for the animal is also a concern for analytical chemists. Experiments can be performed interperitonealy (IP), transdermally (TD), intravenously (IV), orally (PO) or intramuscularly (IM) among others. The PO and 


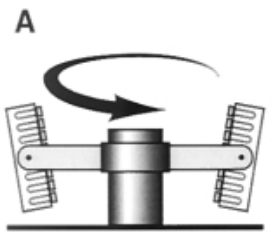

Centrifuge

\section{B}

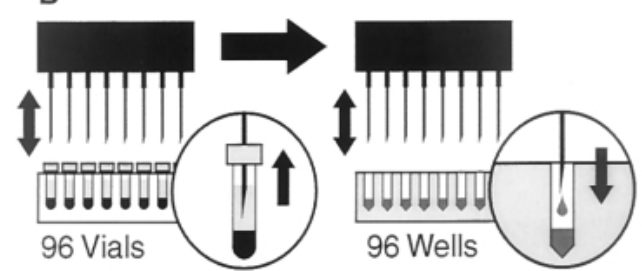

Fig. 3 Plates can be centrifuged and aliquots transferred using robotics; for example, in a protein precipitation step.
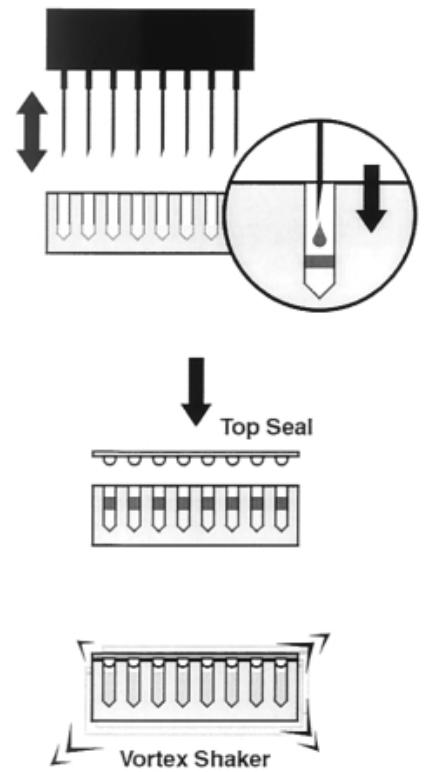

Fig. 4 Extraction solvent can be added and the plate shaken for liquid-liquid extraction.

IV experiments are most common for early PK screening, often to establish oral absorption and to get information about the rate of elimination. There are also cases where it is advantageous to dose the animal with either a bolus or continuous infusion through an indwelling catheter. This too can be fully automated and provides an excellent way to determine the pharmacokinetic clearance parameter, CL. ${ }^{3}$

\subsection{Membrane sampling probes}

One technology that attracted our attention more than twenty years ago is the use of implanted dialysis fibers as passive artificial blood vessels. Today, this takes the form of either microdialysis or ultrafiltration probes. This approach has been widely employed in recent years and is especially useful for small hydrophilic compounds, including glucose, amino acids, neutrotransmitters and some drug substances, ${ }^{4,5}$ Membrane sampling is beneficial in that it does not collect protein (including protein bound drugs) or cellular matter; but these are also limitations. In addition, the concentration recovery of
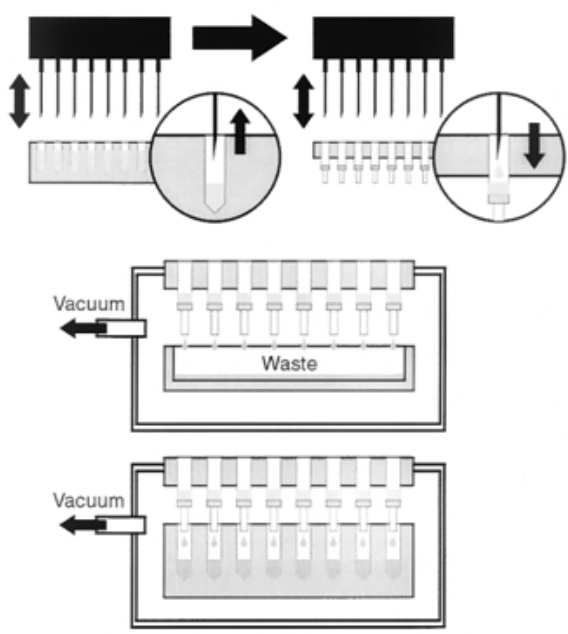

Remove Analytes from SPE Block

Fig. 5 Solid phase extraction (SPE) is often carried out today using plates with robotic loading of samples, washing and finally eluting with a vacuum source.

\section{Warm Nitrogen Blowdown}

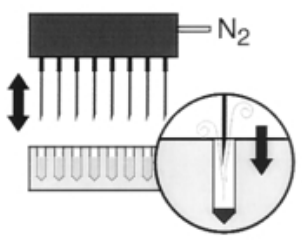

\section{Reconstituted Sample}

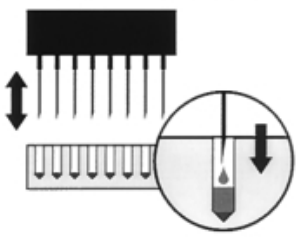

Fig. 6 Evaporating the extracts using warm nitrogen and redissolving in a mobile phase for LC/MSMS is easily done in parallel.

analytes from the extracellular space surrounding a probe is very dependent on physical factors including membrane dimensions, perfusate flow rate, molecular weight and charge. Hydrophobic compounds are problematic in sticking to components of the probe and connecting tubing. Nevertheless, membrane sampling is quite reproducible under given circumstances and is especially useful for following changes in endogenous compounds in response to drugs. Glucose and neurotransmitters are favorite examples.

Figure 7 shows a probe designed for continuous sampling of brain regions in mice and rats. Figure 8 illustrates a schematic for a linear probe used in peripheral tissue. Related designs are available for sampling the vasculature, bile and even bone marrow. The three essential components of such experiments are a syringe pump, a probe and a microfraction collector. Collection rates are on the order of $1 \mu \mathrm{L} / \mathrm{min}$ and, thus, it is rarely practical to collect samples more often than every $5-10$ min. Fortunately, the same refrigerated microfraction collectors used for dialysates can also be used for blood. Coupling to 


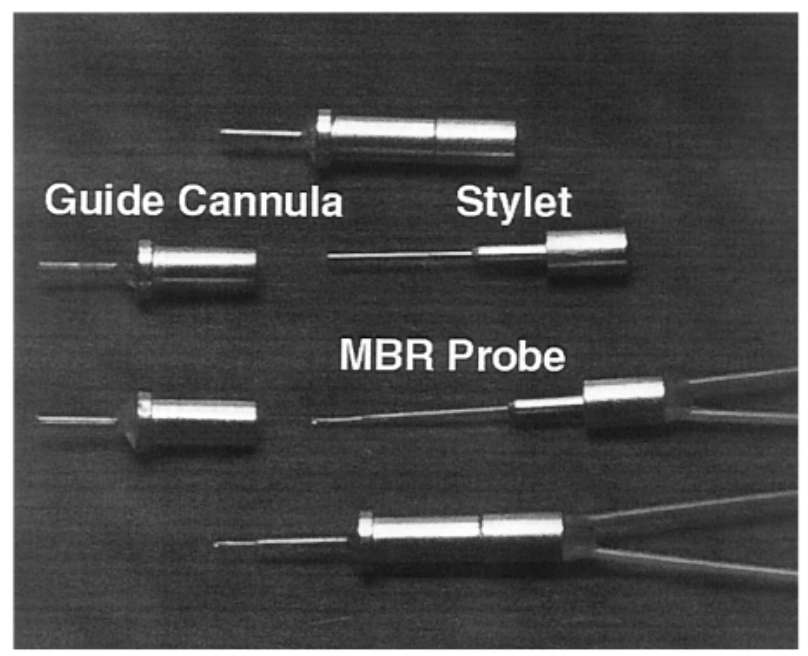

Fig. 7 Microdialysis brain probe (MBR) and accessories for sampling brain regions in mice or rats. A guide cannula is first implanted under anesthesia. Later $(24 \mathrm{~h})$ the stylet is removed and the probe is inserted into the guide. The membrane at the probe tip is $0.22 \mathrm{~mm}$ in outside diameter.

\section{LC/MSMS has been reported by several groups. ${ }^{6,7}$}

\subsection{Automated blood sampling}

Automation of in vivo techniques has not kept pace with in vitro techniques, or with the analytical methods used to process biological samples after collection. Serial blood sampling from rodents can be a logistical challenge when conducted by hand over $12-48 \mathrm{~h}$. The prevailing methodology for manual blood sampling uses basic tools such as syringes and devices for severely restraining animals for tail vein puncture. Intravenous catheters with heparin-saline locks are used for both anesthetized and awake, restrained rats. In other examples, an animal is sacrificed at each time point. These methods all require moving an animal from its cage and might inflict considerable pain and stress. Multiple animals are required for adequate statistics.

Over the last several years, we developed an automated blood sampler (ABS), a robotic system that collects serial blood samples from awake and freely moving rats. ${ }^{8-10}$ This instrument also collects urine and feces, providing additional ADME information from the same subject. The automation adds another parameter by monitoring animal activity during the experiment, providing a look at possible behavioral anomalies associated with the drug much earlier in the screening process. Microdialysis samples can also be collected.

Manual collection of blood from a rat is not simple. Common problems include catheters occluded with blood clots, infections associated with catheter surgery or retrieval, animal damage to catheters, and positional errors caused by shifting or realignment of the catheter tip within the vein. Encountering any of these difficulties may result in loss of a timed sample or at least a significant delay. Animal care and use requirements limit the total amount of blood that can be removed from an individual animal. For these reasons, it is normal to dose several animals and use one for each time point in the PK curve, or perhaps every third time point, depending on the volume of blood taken at each blood draw. Animal-to-animal differences in metabolism occur and can contribute to inconsistencies in the pharmacokinetic data.

Against this background, the automated system generates data

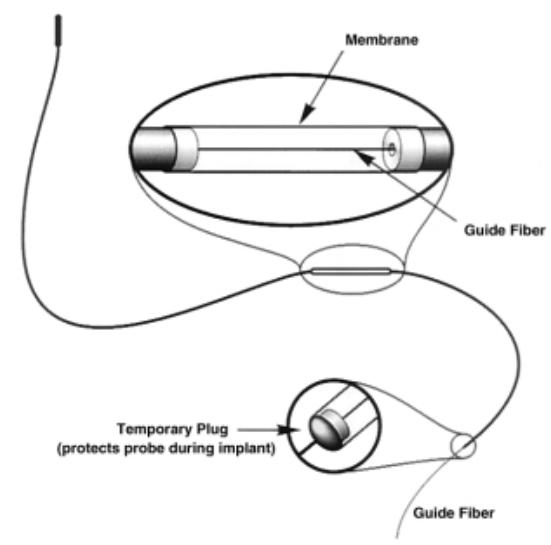

Fig. 8 A linear microdialysis probe for sampling soft peripheral tissue. The membrane length in such probes normally ranges from 5 $-10 \mathrm{~mm}$. The diameter is $0.32 \mathrm{~mm}$.

for the entire PK experiment using one animal. Figure 9 illustrates a 4-animal ABS based on a cart about one meter square. Figure 10 shows a bench top unit for a single animal. Figure 11 outlines schematically how the system takes each sample and prepares for subsequent samples. Each animal has its own independent blood sampling protocol, start/stop time and collection facilities. All functions are controlled by a single notebook computer. Animal damage to catheters is eliminated by affixing the catheter to a tether assembly mounted to a counter-balanced arm that keeps the catheter out of the animal's reach or view. The catheter tubing is protected from being twisted by animal movement through use of the Raturn ${ }^{\circledR}$ system, a movement-responsive cage that monitors the direction of movement and then rotates the cage, and animal within, in the opposite direction. ${ }^{11,12}$ The key innovation here is the total elimination of need for a liquid swivel. Swivels are expensive, prone to leak, and inconvenient to sterilize. They also add unnecessary dead volume and prohibit use of more than one catheter.

Positional errors in catheter placement may occur during repeated handling of the animal, which may cause a catheter tip to shift within the blood vessel. This is unavoidable during manual blood sampling as a rat is removed from its home cage, placed into a restraining device, removed and returned to the cage. Once an animal is installed into the ABS, the only time it is touched again is during a drug administration procedure that necessitates handling (e.g. gavage). Dosing methods such as IV infusions require no contact with the animal. Infusions, both bolus and continuous, can also be programmed using an optional accessory designed in our laboratory. All blood sampling events are conducted hands-off, with the animal free to move at will.

\subsection{Catheter details}

The rat has a sturdy immune system, which makes infection less likely, but not impossible. Sometimes catheters are stored under the skin during shipment of pre-implanted animals, and this may lead to problems with localized infection. For an ABS study, animals are supplied with a removable jacket containing a small pouch to house the externalized catheter tubing. The catheter is protected and the animal has a better opportunity to heal fully. All blood-handling components in the system are provided sterile, including catheters and the tubing set on the instrument itself, to maintain aseptic conditions throughout the 


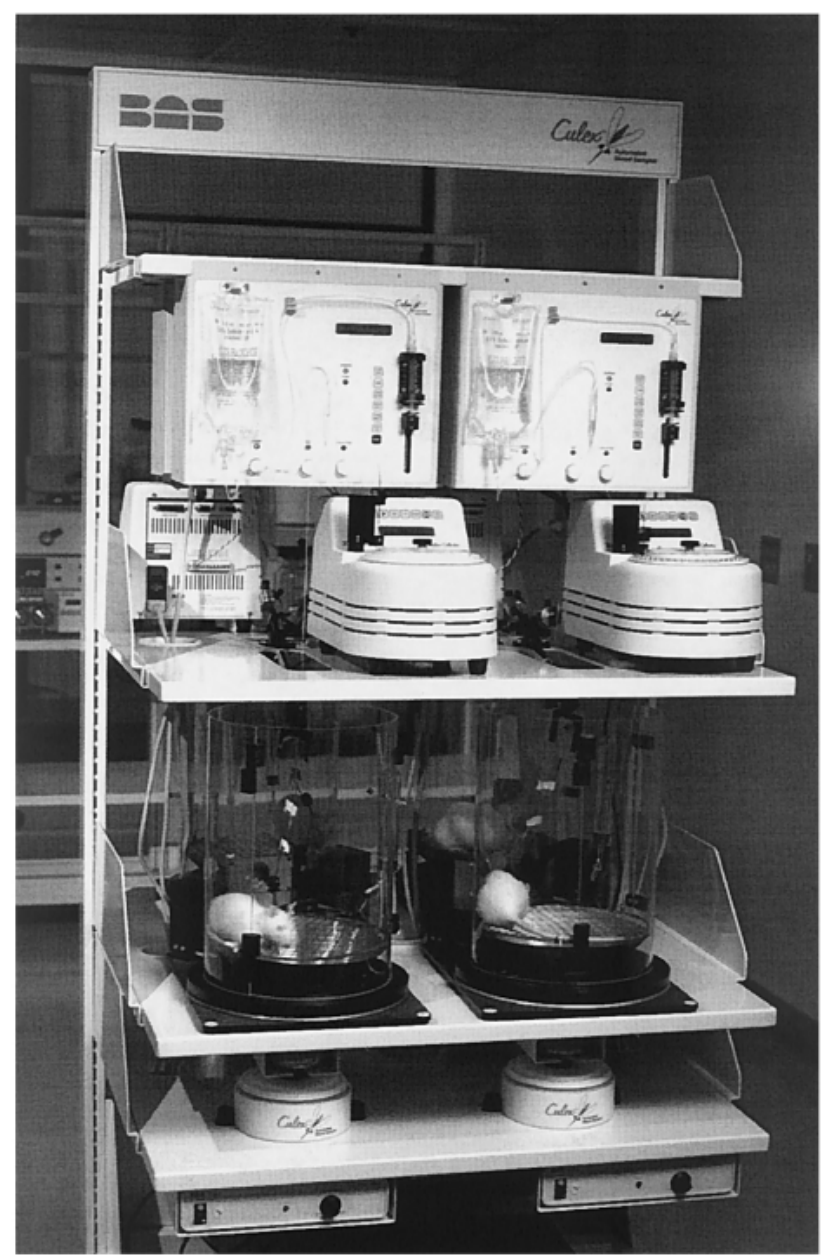

Fig. 9 A robot station for sampling blood, urine and feces while monitoring behavior in four rats. Samples are collected in refrigerated $300 \mu \mathrm{L}$ vials directly transferable to 96-well plates as shown in Fig. 2.

experiment. Our laboratory has established partnerships with several animal suppliers who now supply us animals with the catheters already in place.

Catheters implanted into veins will partially fill with blood that clots and prevents any influx or efflux of fluid thereafter. To prevent this, a "lock" is employed. The locking process fills the catheter completely with a solution to inhibit clotting. Solutions include saline, glycerol, dextrose or combinations of those materials supplemented with the anti-coagulant heparin, usually at concentrations ranging from 10 to 300 units $/ \mathrm{mL}$. A lock is normally installed during surgery to implant the catheter and must be removed prior to sampling blood. If the locking solution does not completely fill the catheter, it is possible for a clot to form at the tip of the catheter and effectively occlude it. The animal requires a recovery period of a day or more after catheter surgery and the heparin locks are replaced daily, involving additional labor. With an $\mathrm{ABS}$, the process of maintaining catheter patency is also automated using proprietary software.

\section{Results}

Since automated blood sampling is primarily being used in commercial pharmaceutical research, relatively little has been

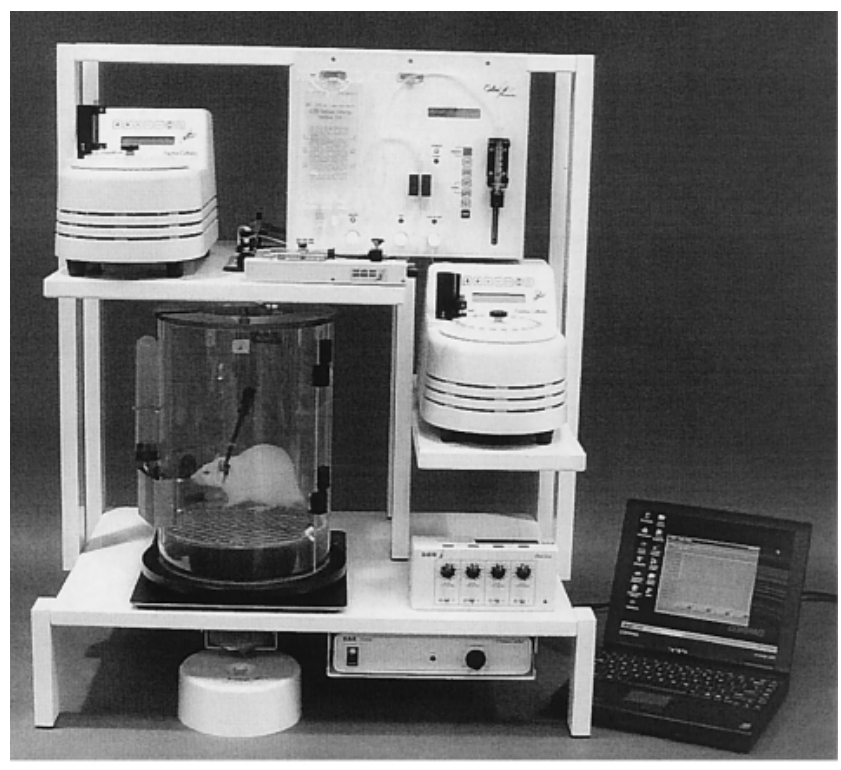

Fig. 10 A benchtop system for simultaneous, automated sampling of blood, microdialysates, urine and feces.

published in the open scientific literature. Traditional pharmacokinetic studies involve intermittent blood sampling and subsequent determination of drug concentrations in blood or plasma. Because of difficulties associated with collecting blood over time from a single rodent, often multiple animals are used to generate a single PK curve, increasing the inter-animal variation. Unlike manual sampling via tail vein or a catheter, automated blood sampling provides a means for blood withdrawal at preprogrammed intervals without the stress associated with handling the animal. ${ }^{8-13}$

Recently, pharmacokinetics studies of the antiepileptic drug carbamazepine (CBZ) and its main metabolite carbamazepine 10,11-epoxide (CBZ-E) were carried out using both automated and manual blood sampling. ${ }^{14}$ Male Sprague Dawley rats were used. Rats were surgically implanted with a jugular (CX-2010, BAS) catheter. Blood sampling was through the jugular catheter. Following surgery, all rats were housed in a metabolic cage (MR-1523, BAS) placed on a Raturn ${ }^{\mathrm{TM}}$, and allowed to recover for one day. Following surgery, rats were given free access to feed and water. Before oral dosing with CBZ, rats were fasted overnight. Rats were dosed with $5 \mathrm{mg} / \mathrm{kg}$ of CBZ. Blood was placed in a vial containing heparin and kept refrigerated until processing. During manual sampling, blood was collected into a syringe and stored in an eppendorf tube in the refrigerator. During sampling with the automated blood sampler, blood was kept in a refrigerated fraction collector. All blood samples were centrifuged and the plasma was obtained. After liquid-liquid extraction by ethyl acetate, the reconstituted residual was injected into the LC/MSMS system. Figure 12 shows the mean plasma concentration-time profiles of CBZ and its main metabolite CBZ-E from seven rats by using automated and manual sampling. It is clearly shown that there is a significant difference for the results obtained between the two methods. It has been recognized that stress of conventional sampling from awake rats drastically influences rodent physiology (blood pressure, blood flow) and biochemistry (stress hormone release, metabolism, even protein expression). ${ }^{15}$ Automated blood sampling permits awake cannulated animals to be sampled stress free (without restraint and human handling). The latter situation provides the most consistent and 

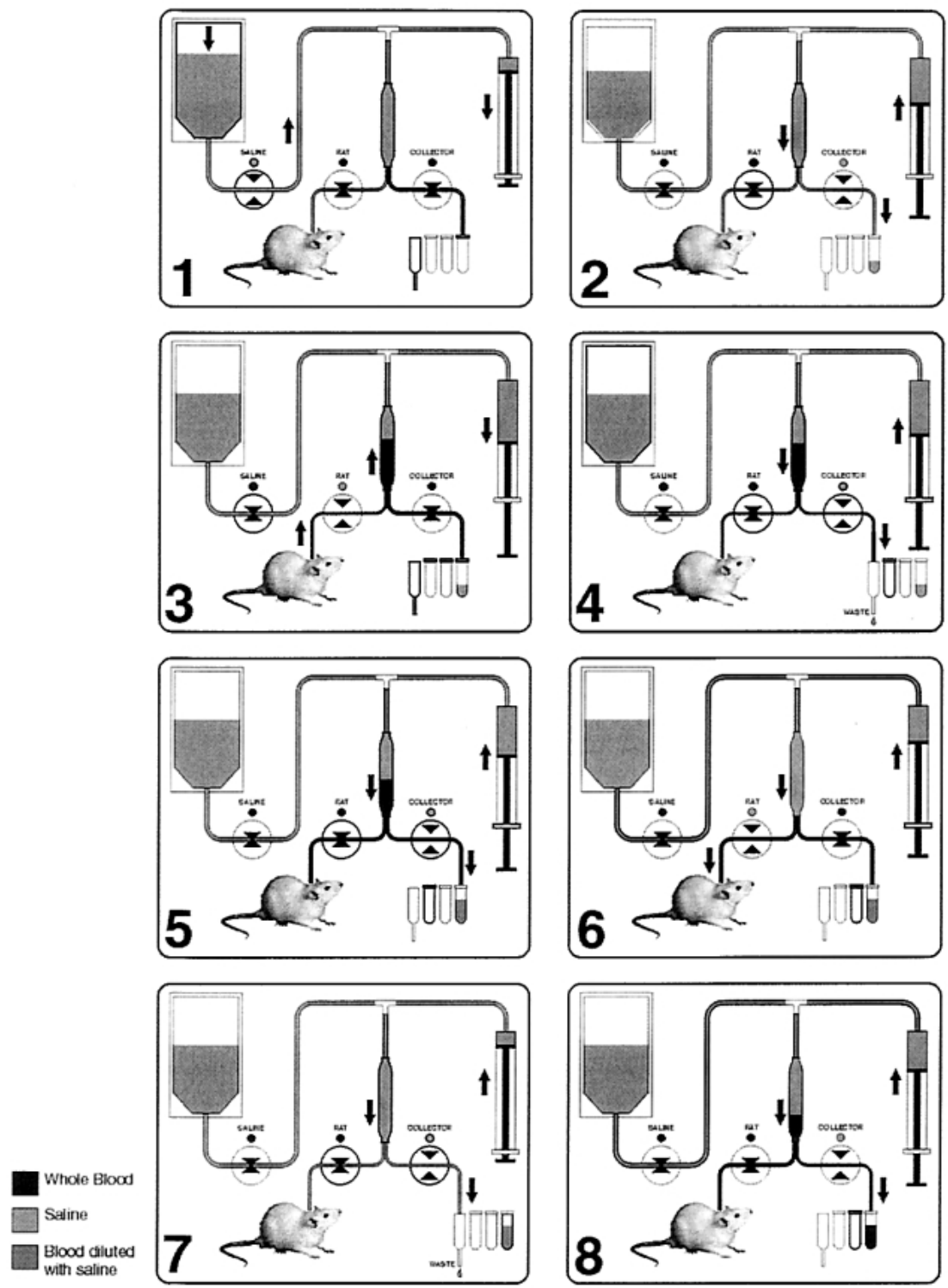

Fig. 11 The ABS system takes each sample and then prepares for subsequent samples. 1 , Sterile tubing set is attached to rat with an implanted catheter. Syringe is partially filled; 2, If diluted blood is desired, saline can be deposited in vials $(300 \mu \mathrm{L})$ automatically; 3 , Blood is drawn from catheter into reservoir; 4, Collector line flushed to waste; 5, Blood collected $(10-250 \mu \mathrm{L})$ is placed in fraction-collector vial; 6, Extra blood and saline returned to restore fluid volume in animal; 7, Collector line flushed with saline; 8, Illustrates Step 5 if Step 2 is skipped to collect whole blood.

Table 1 Mean PK parameters $( \pm \mathrm{SD})$ of carbamazepine and its epoxide metabolite in plasma from rats $(n=7)$

\begin{tabular}{llcccc}
\hline Compound & Treatment & $\begin{array}{c}C_{\max } \\
(\mathrm{ng} / \mathrm{mL})\end{array}$ & $\begin{array}{c}T_{\max } \\
(\mathrm{h})\end{array}$ & $\begin{array}{c}\mathrm{AUC} \\
(\mathrm{ng} \mathrm{h} / \mathrm{mL})\end{array}$ & $\begin{array}{c}\mathrm{CL} \\
(\mathrm{ml} / \mathrm{h})\end{array}$ \\
\hline \multirow{2}{*}{ CBZ } & Manual & $814 \pm 345$ & $0.5 \pm 0.3$ & $1818 \pm 548$ & $1005 \pm 300$ \\
& Culex & $888 \pm 448$ & $0.3 \pm 0.1$ & $1465 \pm 774$ & $1724 \pm 1309$ \\
CBZ-E & Manual & $867 \pm 303$ & $2.9 \pm 2.3$ & $3580 \pm 1155$ & \\
& Culex & $732 \pm 367$ & $2.3 \pm 1.8$ & $3074 \pm 1587$ & \\
\hline
\end{tabular}

meaningful data for PK studies. Table 1 shows PK parameters of CBZ and its epoxide metabolite in plasma from the same rat using two different blood sampling methods. It can be seen clearly that when PK data are obtained from an animal under stress, the normal parameters of $C_{\max }, T_{\max }$, and AUC can all vary substantially.

It is possible to simultaneously collect not only blood samples but also monitor tissue concentrations, for example, from brain or lung. Simultaneous determination of CBZ and its metabolite CBZ-E in rat blood and brain with automated blood sampling and brain microdialysis has been demonstrated. Figure 13 shows the parallel PK profiles of CBZ and CBZ-E in blood and brain as well. This provides another dimension for PK and PD studies during drug discovery and development.

\section{Conclusion}

Automated in vivo sampling with membrane probes and indwelling catheters provides several advantages for both the 

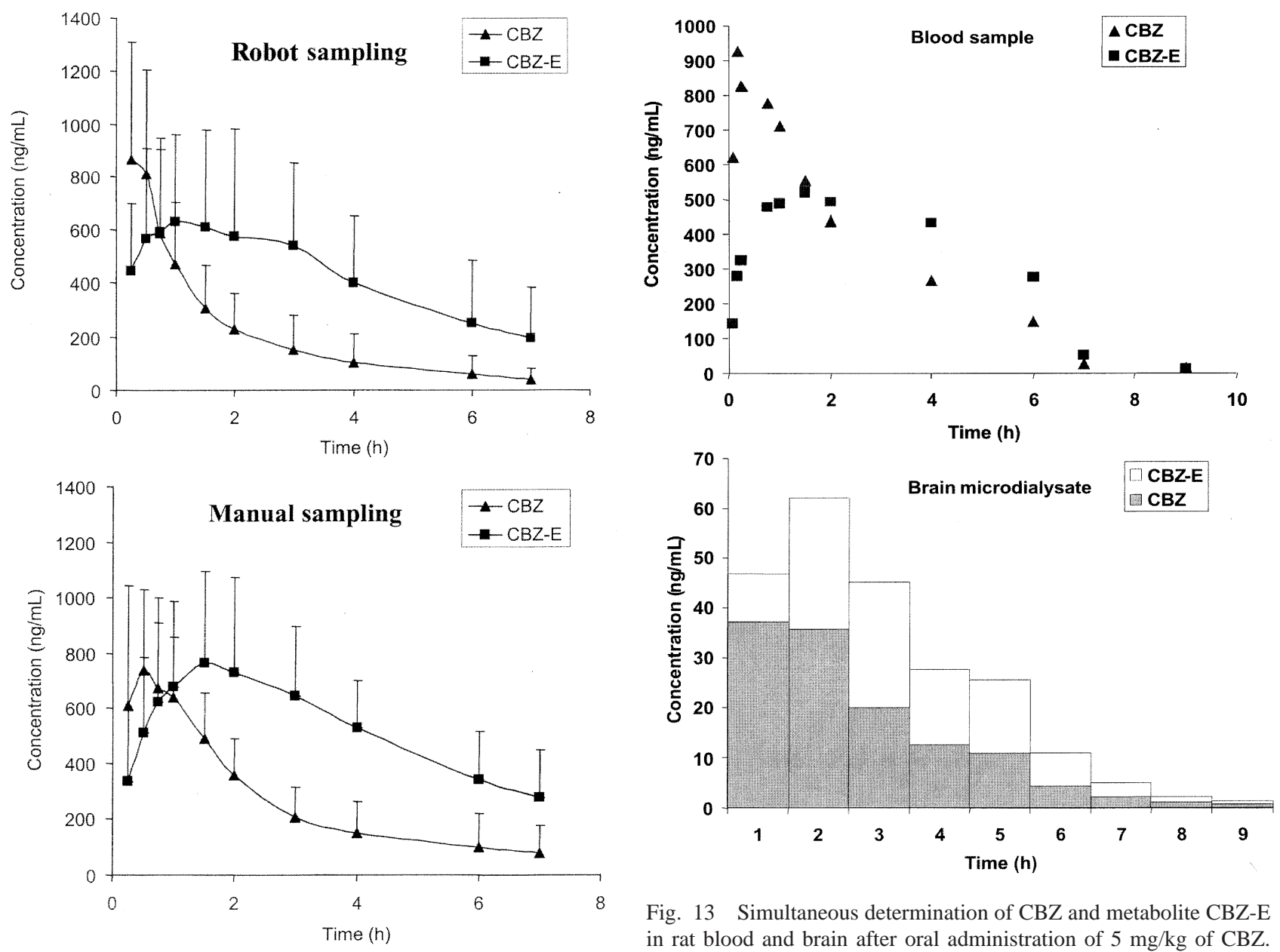

Fig. 12 Mean $( \pm \mathrm{SD})$ plasma concentration-time profiles of CBZ and metabolite CBZ-E in rats $(n=7)$ after oral administration of 5 $\mathrm{mg} / \mathrm{kg}$ of CBZ. Automatic sampling (top) and manual sampling (bottom).

research animal and the scientist. The sampling process is totally painless to the animal. The corresponding reduction in stress improves both data relevance and quality, enabling a reduction in the total number of animals required. Quality assurance is improved because sampling times and volumes are precisely controlled and recorded by software versus manual procedures. Neither an ABS nor microdialysis would be practical were it not for advances in bioanalytical chemistry that permit processing small-volume samples with precision and accuracy. The combination of 96-well plate sample preparation schemes with LC/MSMS is what makes this feasible for virtually all pharmas and Contract Research Organizations (CROs).

\section{References}

1. J. K. Nicholson, J. C. Lindon, and E. Holmes, Xenobiotica, 1999, 11,1181

2. D. G. Robertson, M. D. Reily, R. E. Sligar, D. F. Wells, D. A. Paterson, and T. K. Braden, Toxicol. Sci., 2000, 57, 326.

3. J. E. Riviere, "Comparative Pharmacokinetics: Principles, Techniques and Applications", 1999, Iowa State University Press, 117.

4. M. I. Davies, J. D. Cooper, S. S. Desmond, C. E. Lunte, and S. M. Lunte, Adv. Drug Deliv. Rev., 2000, 45(2-3),
Fig. 13 Simultaneous determination of CBZ and metabolite CBZ-E in rat blood and brain after oral administration of $5 \mathrm{mg} / \mathrm{kg}$ of CBZ. Plasma concentration-time profiles (top) and brain microdialysate concentration-time profiles (bottom). Note that plasma concentrations are instantaneous whereas dialysis concentrations are an average over the 1-h $(60 \mu \mathrm{L})$ collection time for each sample.

169.

5. M. I. Davies, Anal. Chim. Acta, 1999, 379, 227.

6. Y. Zhu, P. S. H. Wong, M. Cregor, J. Gitzen, L. Coury, and P. T. Kissinger, Rapid Commun. Mass Spectrom., 2000, 14 , 1670.

7. Y. Zhu, P. S. H. Wong, Q. Zhou, H. Sotoyama, and P. T. Kissinger, J. Pharm. Biomed. Anal., 2001, 26, 967,

8. Y. X. Zhu, T. H. Huang, C. Cregor, H. Long, C. B. Kissinger, and P. T. Kissinger, J. Chromatogr. B, 2000, $740,129$.

9. H. He, R. Kramp, L. Ramos, and R. Bakhtiar, Rapid Commun. Mass Spectrom., 2001, 15, 1768.

10. H. Long, Y. X. Zhu, M. Cregor, F. F. Tian, L. Coury, C. B. Kissinger, and P. T. Kissinger, J. Chromatogr. B, 2001 , $763,47$.

11. P. T. Kissinger, Current Separations, 2002, 19, 113.

12. www.culex.net

13. F. F. Tian, Y. X. Zhu, H. Long, C. Cregor, F. M. Xie, C. B. Kissinger, and P. T. Kissinger, J. Chromatogr. B, 2002 , $772,173$.

14. Y. X. Zhu, H. Chiang, M. Wulster-Radcliffe, R. Hilt, P. Wong, C. B. Kissinger, and P. T. Kissinger, J. Pharm. Biomed. Anal., submitted.

15. C. B. Kissinger, R. M. Hilt, C. Gunaratna, and Y. X. Zhu, Abstracts, The 6th International ISSX Meeting, Munich, Germany, 2001, 245. 